\title{
New conception in continuum theory of constitutive equation for anisotropic crystalline polymer liquids
}

\author{
Shifang Han \\ Chengdu Institute of Computer Application, Academia Sinica, Chengdu, China; sfh5578@yahoo.com.cn
}

Received 30 May 2010; revised 20 July 2010; accepted 23 July 2010.

\section{ABSTRACT}

A new continuum theory of the constitutive equation of co-rotational derivative type is developed for anisotropic viscoelastic fluid-liquid crystalline (LC) polymers. A new concept of simple anisotropic fluid is introduced. On the basis of principles of anisotropic simple fluid, stress behaviour is described by velocity gradient tensor $F$ and spin tensor $W$ instead of the velocity gradient tensor $D$ in the classic Leslie-Ericksen continuum theory. Analyzing rheological nature of the fluid and using tensor analysis a general form of the constitutive equation of co-rotational type is established for the fluid. A special term of high order in the equation is introduced by author to describe the special change of the normal stress differences which is considered as a result of director tumbling by Larson et al. Analyzing the experimental results by Larson et al., a principle of Nonoscillatory normal stress is introduced which leads to simplification of the problem with relaxation times. The special behaviour of nonsymmetry of the shear stress is predicted by using the present model for LC polymer liquids. Two shear stresses in shear flow of LC polymer liquids may lead to vortex and rotation flow, i.e. director tumbling in the flow. The first and second normal stress differences are calculated by the model special behaviour of which is in agreement with experiments. In the research, the computational symbolic manipulation such as computer software Maple is used. For the anisotropic viscoelastic fluid the constitutive equation theory is of important fundamental significance.

Keywords: Constitutive Equation; Co-rotational Derivative Type; Simple Anisotropic Fluid; Non-Newtonian Fluid; Liquid Crystalline Polymer; Normal Stress Difference; Shear Flow;
Un-symmetry of Shear Stress Tensor; Director Tumbling Effect; Non-Oscillatory Normal Stress

\section{INTRODUCTION}

The rheological behaviour of LC polymer melt and solutions is considerably different from that of the common Polymers [1-3]. Special behaviour of the first and second normal stresses is observed by Baek, Larson, Hudson, Huang [4-6] in the experimental investigation with HPC and PBLG. The experimental results show regions of both positive and negative of the first and second normal stress differences, that is the normal stresses $\sigma_{1}$ and $\sigma_{2}$ change sign two times with variation of shear rates.

The classic Ericksen-Leslie continuum theory [7-12] describes the main features of flow of nematic liquid crystal of low molecular weight or the flow at low shear rates. In research on continuum theory of anisotropic fluids, Green has given attempts to extend basic concept of simple fluid for anisotropic fluid case [13,14]. A simple anisotropic fluid is defined as one for which the stress tensor at a particular particle at time $\tau$ is dependent on the whole history of the deformation gradients $F$ and the whole history of rotation tensor $R$ at the same time. The constitutive equation can be reduced to one which contains only the whole history of the deformation gradients $F$, no history of rotation tensor $R$ in it. The convected constitutive equation of Oldroyd type is well used for the isotropic polymer solutions or melts in Non-Newtonian fluid mechanics, but rarely for the anisotropic LC polymer fluid case. The first attempts were given by Volkov and Kulichikhin for LC polymer fluid $[15,16]$. Using the Maxwell linear equation (1867) for anisotropic liquid crystals and introducing a convected Maxwell model with relaxation and viscosity tensor Vokov and Kulichikhin developed a more simple constitutive equation with non-symmetric shear viscosity. As pointed out by the authors that the constitutive equation is available for the case of small recoverable strains in comparison with the total strains [16]. 
As pointed out by Larson [17], the nematic LC polymer shows director tumbling in shear flow. The experiments have confirmed the tumbling for nematic polymer solutions, but relatively rare in small-molecular nematics. The research of Vokov and Kulichikhin have in addition confirmed that it needs further study on the non-symmetry of the shear stress components in shear flow [16] which may lead to director tumbling in it.

The anisotropic behaviour of LC polymer may be described by the continuum theory. Using convected corotational time derivative, another new approach to continuum theory of the constitutive equation for LC polymer is developed by Han Shifang [1-2,18-22]. The theory was specialized by the LCP-B model [1]. In the equation, both fluid motion and orientational motion are considered. The anisotropic material functions are introduced to describe the anisotropic behaviour of the material. The developed theory is successful to predict special behaviour of the first and second normal stress differences which are in agreement with the experiments [2,4-6].

In the present paper a general continuum theory of constitutive equation is developed which is available for application on both shear and extensional flows of the anisotropic viscoelastic fluids. A new concept of simple anisotropic fluid is introduced. The simple anisotropic fluid is understood as one for which the stress behaviour is assumed to be functional of the whole history of the deformation gradients $F$ and the whole history of spin tensor $W$ instead of rotation tensor $R$ in the Green theory. The present continuum theory is developed to investigate both shear stresses, normal stress differences, and the un-symmetry of the stress tensor for LC polymers. Extending co-rotational Oldroyd fluid B [2] the components of the stress tensor $S_{i j}$ and its co-rotational derivative in it are assumed to be a tensor function of $n_{i}, N_{i}, A_{i j}$ and $W_{i j}$ instead of velocity gradient tensor $D$ in the classic Leslie-Ericksen continuum theory. Using the tensor analysis approach [8-10] and analyzing the physical nature of the fluid, a general form of constitutive equation is constructed for the fluid. A special term $\mu_{1} \omega_{j k} \stackrel{o}{A}_{k i}+\mu_{2} \omega_{i k} \stackrel{o}{A}$ of of high order in equation is introduced by author to describe the special change of the normal stress differences which is considered as a result of director tumbling by Larson et al. Analyzing the experimental results [4-6] a principle of Non-oscillatory normal stress is introduced, which leads to simplification of the problem with relaxation times. The special behaviour of non-symmetry of the shear stress is predicted by using the present model for LC polymer liquids. The present theory is considerably different from the classic Leslie-Ericksen theory and the theory of Volkov and Kulichikhin: 1) the theory is based on the rational mech- anics theory and simple anisotropic fluid which is generally valid for finite deformation case, 2) The constitutive equation is used to study the shear flow to predict the material functions such as apparent viscosity and normal stress differences. The special rheological behaviour observed in experiments is explained by the theory. For shear flow, two shear stresses and two apparent viscosities are obtained which are different from the isotropic fluid.

\section{SIMPLE ANISOTROPIC FLUID}

The "Simple fluid" is a fundamental concept which is based on the theory for modern non-Newtonian fluid mechanics; it is generally valid for isotropic fluid. The "principle of objectivity of material properties" introduced by Noll (1958) is well used to construct constitutive equation in non-Newtonian fluid mechanics and rheology. The simple fluid in sence of Noll is a great significance in construction of constitutive equation theory for isotropic non-Newtonian fluids. But as pointed out by Tanner [23], it is easy to construct physical systems where this principle does not hold. For example it does not hold for a dilute suspension of spheres when the microscale Reynolds number is not negligible [23]. Zahorski noted [24] that the requirement of invariance with respect to the reference frame in considerations involving some fields may prove to be too restrictive. The principle may also be too restrictive for anisotropic fluids! Th- erefore, the concept of simple fluid should be improved further for the special case - anisotropic viscoelastic fluid.

According to the polar decomposition theorem, the deformation gradient $F$ can be decomposed as

$$
\begin{gathered}
F(t)=R(t) U(t), F(\tau)=R(\tau) U(\tau), \\
R(t) R^{T}(t)=R(\tau) R^{T}(\tau)=I,
\end{gathered}
$$

where $R(t)$ is a orthogonal rotation tensor, $U(t)$ is symmetric positive definite tensor. A spin tensor can be defined in terms of $R(t)$

$$
\dot{R}(t)=W(t) R(t), \dot{R}(\tau)=W(\tau) R(\tau)
$$

where $W(t)+W^{T}(t)=0$.

An angular velocity of the superposed rotation is defined as

$$
\dot{Q}(t)=\Omega(t) Q(t), Q(t) Q^{T}(t)=I
$$

When the reference frame is changed the spin tensor is changed by

$$
W^{*}(t)=Q(t) W(t) Q^{T}(t)+\Omega(t)
$$

It can be seen from Eq. (4) that the spin tensor $W(t)$ measured with respect to fixed coordinate system at time $t$ does not satisfy principle of objectivity of material pr- 
operties; the tensor is non-objective. The concept of superposed rotation is introduced. Let the observer is attached to the rotating particle of fluid, i.e. in co-rotational coordinate system. The relationship between the spin tensor $W_{s}(t)$ measured with respect to the fixed reference frame and the spin tensor $W_{c}(t)$ measured with respect to the co-rotational reference frame is given as

$$
W_{s}(t)=W_{c}(t)+Q^{T}(t) \Omega^{T}(t) Q(t)
$$

It is easily proved that the spin tensor $W_{c}(t)$ is also anti-symmetric

$$
W(t)_{c}+W_{c}^{T}(t)=0
$$

Substitution of (5) into (4) yields

$$
W^{*}{ }_{c}(t)=Q(t) W_{c}(t) Q^{T}(t)
$$

The new spin tensor $W_{c}(t)$ measured with respect to the co-rotational reference frame is objective. The concept of simple anisotropic fluid was defined first by Green $[13,14]$ in which the extra stress tensor is expressed by a functional of defomation gradient $F_{\tau}(\tau-s)$ and rotation tensor $R(\tau-s)$. A concept of superposed rigid body rotations is introduced by Green which leads to the following conclusion that the rotation tensor does not affect stress, apart from orientation, i.e. invariance of the equations with respect to superposed rigid rotations. The conclusions of Green are only valid for the nematic liquid crystal of low molecular weight or the flow at low shear rates with any orientation. The nematic liquid crystalline polymer shows director tumbling in shear flow which is confirmed by experiments for nematic polymer solutions [17]. Using the Maxwell linear equation (1867) for anisotropic liquid crystals, non-symmetric shear stresses in shear flow were founded by Vokov and Kulichikhin for LC polymer liquids $[15,16]$ which may be a cause of rotation motion in the flow.

According to the previous discussions it is neccessary to define a new concept of simple anisotropic fluid for the liquid crystalline polymers, which is a basic point of the present investigation. Instead of rotation tensor $R(t)$, a spin tensor $W_{s}$ in constitutive equation measured with respect to fixed coordinates is expressed by a sum of spin tensor $W_{c}$ measured with respect to corotational coordinates and co-rotational tensor term as given by (5). The simple anisotropic fluid is defined as one for which the stress tensor at a particular particle is a functional of the whole history of the deformation gradient $F$ and the whole history of spin tensor $W$ measured with respect to the co-rotational coordinate system.

$$
T={\underset{0}{\infty}}_{0}^{\infty}\left\{F_{\tau}(\tau-s), W(\tau-s)\right\}
$$

where $W$ is defined by (5).

\section{PRINCIPAL CONCEPTS FOR CONSTITUTIVE EQUATION}

In construction of continuum theory of constitutive equation for the LC polymer-anisotropic viscoelastic fluids, the following principal concepts are introduced:

1) A concept of anisotropic simple fluid is introduced. According to the new definition the stress is dependent on the whole history of deformation gradient and the whole history of spin tensor measured with respect to co-rotational coordinate system.

2) The constitutive equation contains both contributions due to the orientational motion of director and hydrodynamic motions of fluid, to describe anisotropic effects of LC polymer [18-22]. The stress tensor is considered as a functional of the deformation tensors and tensors composed of the director vector and its derivative. According to statistic physics, the macroscopic magnitudes are considered as an average of microscopic values.

3) Because the nematic LC polymer solution is also viscoelastic fluid, the constitutive equation of co-rotational Oldroyd fluid B is an initial point in constructing the equation theory for anisotropic viscoelastic fluid. Constitutive equation for anisotropic viscoelastic fluid can be constructed by generalizing co-rotational Oldroyd fluid $\mathrm{B}$ [2].

The Oldroyd fluid B of upper-convected derivative type is well used for isotropic non-Newtonian fluid mechanics. The Oldroyd fluid B with upper-convected derivative is extended to the case of co-rotational time derivative developed by Shifang Han [21,22]

$$
S_{i j}+\lambda_{0} \stackrel{o}{S}_{i j}=\eta_{0} A_{i j}+\mu_{0} \stackrel{o}{A}_{i j}
$$

where $\lambda_{0}$-isotropic relaxation time; $\eta_{0}$-isotropic limiting viscosity. the $S_{i j}$-components of the extra- stress tensor, $A_{i j}$-components of the first Rivlin- Ericksen tensor, the top circle "o" denotes the contrav- ariant components of co-rotational time derivative defined as

$$
\stackrel{\circ}{S^{i j}}=\frac{\partial S^{i j}}{\partial t}+v^{m} S_{, m}^{i j}-\omega_{k}^{i} S^{k j}-\omega_{k}^{j} S^{k i}
$$

For anisotropic fluid, a generalized Maxwell equation is given as [15]

$$
\lambda_{i j k l} \frac{d S_{k l}}{d t}+S_{i j}=\eta_{i j k l} \varepsilon_{k l}
$$

where the relaxation time tensor $\lambda_{i j k l}$ is defined by

$$
\eta_{i j k l}=\lambda_{i j k l} G_{i j k l}
$$

Eq.11 describes the linear anisotropic viscoelastic fluid behavior. 
In the Leslie-Ericksen continuum theory [7-12], the hydrodynamic components of the stress tensor $S_{i j}$ are assumed to be a tensor function of $n_{i}, N_{i}$ and $D_{i j}$, the full deformation history is described only by the symmetric part of the velocity gradient $D$, i.e. the rate tensor $D_{i j}$ are symmetric. This is a limitation of the Leslie-Ericksen theory. According to new definition of anisotropic viscoelastic simple fluid instead of velocity gradient tensor $D$ in the classic theory, the stress tensor is described by $1^{\text {st }}$ Rivlin-Ericksen tensor $A$ and spin tensor $W$ for the solution and the fluid. Extending the general principle in constructing constitutive equation by Truesdell [25] and Ericksen [8] and generalizing constitutive equation of co-rotational Oldroyd fluid B $[19,20]$ and the generalized Maxwell equation, the stress components and those co-rotational derivative are assumed to be of functional of $n_{i}, N_{i}, A_{i j}$ and $W_{i j}$, a general form of the constitutive equation of the fluid is assumed to be of

$$
S_{i j}+\lambda_{i j k l} \stackrel{o}{S} k l=\mu \stackrel{o}{A_{i j}}+\Omega_{i j}^{1}\left[A_{i j}, \omega_{i j}, n_{i}, N_{i}, \beta_{j}\right]
$$

where the $\lambda_{s}, \beta_{j}$ are material constants, $A_{i j}-$ omponents of the first Rivlin-Ericksen tensor, $\omega_{i j}-$ components of spin tensor $W, N_{i}=\dot{n_{i}}-\omega_{i k} n_{k}$.

For anisotropic viscoelastic fluid-LC polymer melt and solution the stress tensor is an un-symmetric one. The anisotropy in elasticity of LC polymers leads to an un-symmetry of the stress tensor. The rotation of the director vector is a source of dissipation in the nematic liquid even in the absence of flow [16]. The stress relationship derived from the Ossen integral equation shows that for the nematic fluid the orientational motion of the director vector characterized by the director surface body stress and intrinsic director body force, which leads to un-symmetry in stress tensor.

The first Rivlin-Ericksen tensor $A_{i j}$ express deformation history due to the normal-symmetric part of the deformation velocity gradient in the fluid, the spin tensor $W_{i j}$ express deformation history due to the un-symmetric part of deformation velocity gradient in the fluid. However the un-symmetry of the stress tensor is determined by the un-symmetry of the shear stress components. It does not have principal influence on the normal stress differences which is of completely symmetric. The stress tensor can be split into two parts: symmetric and un-symmetric

$$
S_{i j}=S_{i j}^{n}+S_{i j}^{s}
$$

where " $n$ " denotes normal-symmetric part, " $s$ "denotes shear un-symmetric part. The functional $\Omega_{i j}$ in (13) is correspondently split into symmetric and un-symmetric too.

For the normal-symmetric part of the stress tensor the general form of the constitutive equation is proposed as

$$
\begin{aligned}
S_{i j}^{n}+\lambda_{i j k l}{\stackrel{o}{S_{k l}}} & =\Psi_{i j}\left[A_{i j}, \stackrel{o}{A_{i j}}, n_{i}, N_{i}, \beta_{j}\right] \\
& +\mu_{1} \omega_{j k} \stackrel{o}{A_{k i}}+\mu_{2} \omega_{i k} \stackrel{o}{A_{k j}}
\end{aligned}
$$

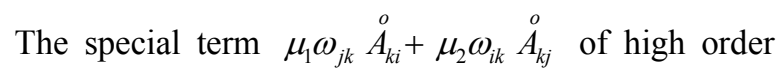
in Eq.15 is introduced to describe the special change of the normal stress differences which is considered as a result of director tumbling effect by Larson et al. [4-6,17].

For the shear un-symmetric part of the stress tensor the general form of the constitutive equation is proposed as

$$
\begin{gathered}
S_{i j}^{s}+\lambda_{k l}^{s} \stackrel{o}{S_{i j k}^{s}}=\Phi_{i j}\left[\omega_{i j}, n_{i}, N_{i}, \gamma_{j}\right], \\
i \neq j, k=1,2,3
\end{gathered}
$$

In Eqs.15 and 16 the relaxation time tensor components $\lambda_{i j k l}^{n}$ and $\lambda_{k l}^{s}$ are introduced for normal-symmetric and shear un-symmetric stresses respectively.

\subsection{Normal Symmetric Part}

Attention will be restricted to which the tensor functionnal are linear tensor functions in the variables $n_{i}, N_{i}$, $A_{i j}$ and $\omega_{i j}$. Thus, for the normal-symmetric Eq.16 one has

$$
\Psi_{i j}=C_{i j}^{0}+C_{i j k}^{1} N_{k}+C_{i j k s}^{2} A_{k s}
$$

The transversely isotropic tensors with respect to direction $n_{i}$ were studied by Smith, Rivlin and Ericksen $[7,12]$ that any such tensor is expressible as a linear combination of outer products formed from tensors $n_{i}$ and $\delta_{i j}-n_{i} n_{j}$ or equivalently

$$
n_{i}, \delta_{i j}
$$

For the anisotropic viscoelastic liquid,the coefficients in (17) are transversely isotropic considering

$N_{i} n_{i}=A_{i i}=0, \varepsilon_{11} \delta_{k s} A_{k s}=0, \varepsilon_{0}=0$, as $A_{i j}$ being chosen arbitrarily, $\varepsilon_{1}=0 \quad \varepsilon_{6}=0$ one can obtain

$$
\begin{aligned}
S_{i j}^{n}+\lambda_{i j k l} S_{k l}^{n}= & \mu_{0} \stackrel{o}{A}_{i j}+\mu_{1} \omega_{j k} \stackrel{o}{A}_{k i}+\mu_{2} \omega_{i k} \stackrel{o}{A_{k j}} \\
& +\varepsilon_{13} A_{i j}+\varepsilon_{14} A_{k s} n_{k} n_{s} n_{i} n_{j} \\
& +\varepsilon_{15} n_{j} n_{k} A_{k i}+\varepsilon_{16} n_{i} n_{k} A_{k j} \\
& +\varepsilon_{3} n_{j} N_{i}+\varepsilon_{4} n_{i} N_{j}
\end{aligned}
$$

where $\varepsilon_{15}=\varepsilon_{7}+\varepsilon_{8}, \varepsilon_{16}=\varepsilon_{9}+\varepsilon_{10}$.

For the case of anisotropy of most general form $[15,16]$ because of symmetry of stress tensor the relaxation time tensor $\lambda_{i j k l}$ has following property

$$
\lambda_{i j k l}=\lambda_{j i k l}=\lambda_{i j l k,} \quad \lambda_{i j l m}=\lambda_{l m i j},
$$

The Eq.18 can be rewritten as

$$
S_{i j}^{n}+\lambda_{i j k l} \stackrel{o}{S_{k l}^{n}}=\eta A_{i j}+\mu_{0} \stackrel{o}{A_{i j}}+\mu_{1} \omega_{j k} \stackrel{o}{A_{k i}}
$$




$$
\begin{aligned}
& +\mu_{2} \omega_{i k} \stackrel{o}{A_{k j}}+\beta_{1}^{*} A_{k s} n_{k} n_{s} n_{i} n_{j} \\
& +\beta_{2}^{*} n_{j} n_{k} A_{k i}+\beta_{3}^{*} n_{i} n_{k} A_{k j} \\
& +\beta_{4}^{*} n_{j} N_{i}+\beta_{5}^{*} n_{i} N_{j}
\end{aligned}
$$

where

$$
\begin{gathered}
\eta=\varepsilon_{12}, \\
\beta_{1}^{*}=\varepsilon_{14}, \beta_{2}^{*}=\varepsilon_{15}, \beta_{3}^{*}=\varepsilon_{16}, \beta_{4}^{*}=\varepsilon_{3}, \beta_{5}^{*}=\varepsilon_{4} .
\end{gathered}
$$

The molecular density in the Ossen integral equation can be considered as a small magnitude, and the molecular inertia is neglected, one can obtain from director transport equation $[1,2]$

$$
-m n_{i}+\gamma_{1}^{*} N_{1}+\gamma_{2}^{*} n_{j} A_{j i}=0
$$

Because of Eq.(21) the co-rotational term $N_{i}$ in Eq.(18) can be expressed by $n_{i}, A_{i j}$, and the constitutive Eq.18 is reduced to the following form

$$
\begin{aligned}
S_{i j}+\lambda_{i j k l} \stackrel{o}{S}_{k l}^{n}= & \eta A_{i j}+\mu_{0} \stackrel{o}{A}_{i j}+\mu_{1} \omega_{j k} \stackrel{o}{A_{k i}} \\
& +\mu_{2} \omega_{i k} \stackrel{o}{A_{k j}}+\beta_{1} n_{i} n_{j} n_{k} n_{s} A_{k s} \\
& +\beta_{2} n_{i} n_{k} A_{k j}+\beta_{3} n_{j} n_{k} A_{i k}
\end{aligned}
$$

\subsection{Shear Un-symmetric Part}

For the shear un-symmetric partial Eq.16 one has

$$
\Phi_{i j}=E_{i j k}^{1} N_{k}+E_{i j k s}^{2} \omega_{k s}
$$

The coefficient tensors $E_{i j k}^{1}, E_{i j k s}^{2}$ are expressible as a linear combination of outer products formed by $n_{i}, \delta_{i j}$. For the shear un-symmetric part, the partial Eq.16 can be reduced to the following form [2]

$$
\begin{aligned}
S_{i j}^{s}+\lambda_{k l}^{s} S_{i j k}^{o}= & \chi_{1} n_{j} n_{s} \omega_{i s}+\chi_{2} n_{i} n_{s} \omega_{j s} \\
& +\chi_{3} \omega_{i j}+\chi_{4} n_{i} n_{j} n_{k} n_{s} \omega_{k s}
\end{aligned}
$$

where

$$
\chi_{1}=\gamma_{7}-\gamma_{8}, \chi_{2}=\gamma_{9}-\gamma_{10}, \chi_{3}=\gamma_{13}, \varphi_{4}=\gamma_{14}
$$

In Eqs.22 and $24 \lambda_{i j k l}$-components of anisotropic relaxation time tensor, dimension of which is $[s] ; \eta$, $\beta_{1}, \beta_{2}, \beta_{3}$-anisotropic viscosities being influence of the orientational motion on the viscosity; $\mu$-anisotropic retardation time ; $\eta, \beta, \beta_{1}, \beta_{2}, \beta_{3}, \chi_{1}, \chi_{2}$, $\chi_{3}, \chi_{4}-[$ Pas $], \mu-\left[\mathrm{Pas}^{2}\right]$.

In research on rheology and non-Newtonian fluid mechanics for a new constitutive equation, the material functions of the typical flows, such as shear flow and extensional flow, are considered to proof the reasonableness and availability of the theory. Now axial-symmetric shear flow will be studied to show the reasonableness and availability of present constitutive theory. The cylindrical coordinate system $(z, r, \phi)$ is used. The velocity field and the director field are given as

$$
V=(u, 0, w), \quad n=\left(n_{r}, 0, n_{z}\right)
$$

For the velocity field (25) one has

$$
\begin{aligned}
& \omega_{r z}=\frac{1}{2}\left(\frac{\partial u}{\partial z}-\frac{\partial w}{\partial r}\right), \omega_{z r}=\frac{1}{2}\left(\frac{\partial w}{\partial r}-\frac{\partial u}{\partial z}\right) . \\
& \omega_{r r}=\omega_{\phi \phi}=\omega_{z z}=0 .
\end{aligned}
$$

For the velocity field (25) co-rotational time derivative components of extra stress components are calculated. For velocity field (25) and the normal-symmetric part of the stress and by using expressions for co-rotational derivative the constitutive Eq.22 can be reduced to equations in stress components.

The property of symmetry for the relaxation time tensor $\lambda_{i j l m}$ (19) was used. Using the definition of co-rotational derivative, the constitutive Eq.22 can be reduced. The stress process is assumed to be time-independent; the constitutive Eq. (22) is finally reduced as following

$$
\begin{aligned}
S_{r r} & -2 \omega_{r z}\left(\lambda_{11}^{n}-\lambda_{13}\right) S_{r z}+\lambda_{12}^{n} \omega_{r z}\left(S_{r r}-S_{z z}\right) \\
= & 2 \eta \frac{\partial u}{\partial r}-2 \mu_{0} \omega_{r z} A_{r z}+\left(\mu_{1}+\mu_{2}\right)\left(\omega_{r z}\right)^{2}\left(A_{r r}-A_{z z}\right) \\
& +\beta_{1} n_{r}^{2}\left(n_{r}^{2} A_{r r}+n_{z}^{2} A_{z z}+2 n_{r} n_{z} A_{r z}\right) \\
& +\left(\beta_{2}+\beta_{3}\right) n_{r}\left(n_{r} A_{r r}+n_{z} A_{r z}\right) \\
S_{z z} & -2 \omega_{r z}\left(\lambda_{12}^{n}-\lambda_{23}^{n}\right) S_{r z}+\lambda_{22}^{n} \omega_{r z}\left(S_{r r}-S_{z z}\right) \\
= & 2 \eta \frac{\partial w}{\partial r}+2 \mu_{0} \omega_{r z} A_{r z}-\left(\mu_{1}+\mu_{2}\right)\left(\omega_{r z}\right)^{2}\left(A_{r r}-A_{z z}\right) \\
& +\beta_{1} n_{z}^{2}\left(n_{r}^{2} A_{r r}+n_{z}^{2} A_{z z}+2 n_{r} n_{z} A_{r z}\right) \\
& +\left(\beta_{2}+\beta_{3}\right) n_{z}\left(n_{r} A_{r r}+n_{z} A_{r z}\right) \\
& S_{r z}-2 \omega_{r z}\left(\lambda_{13}^{n}-\lambda_{33}^{n}\right) S_{r z}+\lambda_{23}^{n} \omega_{r z}\left(S_{r r}-S_{z z}\right) \\
= & \eta\left(\frac{\partial u}{\partial z}+\frac{\partial w}{\partial r}\right)+\mu_{0} \omega_{r z}\left(A_{r r}-A_{z z}\right)+2\left(\mu_{1}-\mu_{2}\right) \omega_{r z}^{2} A_{r z} \\
& +\beta_{1} n_{r} n_{z}\left(n_{r}^{2} A_{r r}+n_{z}^{2} A_{z z}+2 n_{r} n_{z} A_{r z}\right) \\
& +\beta_{2} n_{r}\left(n_{r} A_{r z}+n_{z} A_{z z}\right)+\beta_{3} n_{z}\left(n_{r} A_{r r}+n_{z} A_{r z}\right)
\end{aligned}
$$

It is an additional director transport equation derived from conservation equation of director (Ossen's equation) $[1,2,11,21]$.

$$
-\rho_{1} \frac{\partial F}{\partial n_{i}}-\rho_{1} \frac{\partial}{\partial x_{j}}\left(\frac{\partial F}{\partial n_{i, j}}\right)=-\gamma n_{i}+\gamma_{1} N_{i}+\gamma_{2} n_{j} A_{j i} .
$$

Neglecting inertia and gravity forces the director transport equation is reduced to

$$
\frac{\partial n_{i}}{\partial t}+v_{j} n_{i, j}=\omega_{i k} n_{k}+\lambda_{b}\left(n_{k} A_{k i}-n_{i} n_{k} n_{l} A_{k l}\right) .
$$


For the axial symmetric problem one has the following relationship

$$
n_{r}^{2}+n_{z}^{2}=1 \text {, }
$$

two transport equations are depedent, whereas only one equation is independent. For the Poiseuille flow

$$
\frac{\partial \theta(r, t)}{\partial \mathrm{t}}=-\left[\frac{1}{2}+\lambda_{b}\left(1-2 \cos ^{2} \theta(r, t)\right)\right] \frac{\partial w}{\partial r} .
$$

For statinary state there is a constant director vector for shear flow

$$
\begin{aligned}
& n_{z}{ }^{0}=\cos \theta_{0}= \pm \sqrt{\frac{2 \lambda_{b}+1}{4 \lambda_{b}}}, \\
& n_{r}{ }^{0}=\sin \theta_{0}= \pm \sqrt{\frac{2 \lambda_{b}-1}{4 \lambda_{b}}} .
\end{aligned}
$$

For the unsteady state the shear flow can transit to tumbling [2].

\section{NORMAL STRESS DIFFERENCES FOR SHEAR FLOW}

The rheological behaviour of the normal stress differences are specially interested. The flow of the LC polymer fluid in circular tube with radius of $\mathrm{R}$ is studied. The Poiseuille flow is such a typical shear flow of the fluid. For the flow components of velocity and director vector are expressed by

$$
\begin{aligned}
& v_{z}=w(r), \quad v_{r}=v_{\phi}=0 \\
& n_{r}=\sin \theta(r), n_{z}=\cos \theta(r)
\end{aligned}
$$

where the $\theta$ is the orientational angle of the director vector. which is a function only for $r$ only. The $1^{\text {st }}$ Rivlin-Ericksen tensor $A$ and the spin tensor $W$ are given as

$$
A=\left|\begin{array}{ccc}
0 & 0 & A_{r z} \\
0 & 0 & 0 \\
A_{r z} & 0 & 0
\end{array}\right|, W=\left|\begin{array}{ccc}
0 & 0 & \omega_{r z} \\
0 & 0 & 0 \\
\omega_{z r} & 0 & 0
\end{array}\right|
$$

The components of $1^{\text {st }}$ Rivlin-Ericksen tensor and spin tensor are given by

$$
A_{r z}=\frac{\mathrm{d} \mathrm{w}}{\mathrm{d} \mathrm{r}}=\dot{\gamma}, \quad \omega_{r z}=-\frac{1}{2} \frac{d w}{d z}=-\frac{1}{2} \dot{\gamma},
$$

For the velocity and director vector fields (31) and (32), the constitutive equations in components Eqs.28$\mathbf{3 0}$ are reduced to the following form

$$
\begin{gathered}
S_{r r}-2 \lambda_{1} \omega_{r z} S_{r z}+\lambda_{4} \omega_{r z} \sigma \\
=\mu_{0} \dot{\gamma}^{2}+\sin \theta \cos \theta\left[2 \beta_{1} \sin ^{2} \theta+\left(\beta_{2}+\beta_{3}\right)\right] \dot{\gamma}
\end{gathered}
$$

$$
\begin{aligned}
& S_{z z}-2 \lambda_{2} \omega_{r z} S_{r z}+\lambda_{5} \omega_{r z} \sigma \\
& =-\mu_{0} \dot{\gamma}^{2}+\sin \theta \cos \theta\left[2 \beta_{1} \cos ^{2} \theta+\left(\beta_{2}+\beta_{3}\right)\right] \dot{\gamma} \\
& S_{r z}-2 \lambda_{3} \omega_{r z} S_{r z}+\lambda_{6} \omega_{r z} \sigma \\
& =\eta \dot{\gamma}+\mu_{*} \dot{\gamma}^{3}+\left[2 \beta_{1} \sin ^{2} \theta \cos ^{2} \theta\right. \\
& \left.\quad+\left(\beta_{2} \sin ^{2} \theta+\beta_{3} \cos ^{2} \theta\right)\right] \dot{\gamma}
\end{aligned}
$$

where the following relaxation times and normal stress difference were introduced

$$
\begin{aligned}
& \lambda_{1}=\lambda_{11}^{n}-\lambda_{13}^{n}, \quad \lambda_{2}=\lambda_{12}^{n}-\lambda_{23}^{n}, \\
& \lambda_{3}=\lambda_{13}^{n}-\lambda_{33}^{n}, \quad \lambda_{4}=\lambda_{12}^{n}, \\
& \lambda_{5}=\lambda_{22}^{n}, \lambda_{6}=\lambda_{23}^{n}, \sigma=S_{r r}-S_{z z}, \\
& \mu_{*}=\frac{1}{2}\left(\mu_{1}-\mu_{2}\right)
\end{aligned}
$$

Solving Eqs.34-36 by the computational symbolic manipulation, such as Maple, general analytical expressions are obtained by the constitutive equation for the first and second shear stresses and the normal stress differences .

Case 1: Director is parallel to flow direction $\sin \theta=0$, $\cos \theta=1$ The first and second normal stress differences are given as

$$
\begin{aligned}
& \sigma_{1}=\frac{2 \dot{\gamma}^{2}}{2+\left[\left(\lambda_{1}-\lambda_{2}\right) \lambda_{6}+\left(\lambda_{5}-\lambda_{4}\right) \lambda_{3}\right] \dot{\gamma}^{2}+\left(2 \lambda_{3}-\lambda_{4}+\lambda_{5}\right) \dot{\gamma}} \\
& \times\left[\left(\lambda_{1}-\lambda_{2}\right) \mu^{*} \dot{\gamma}^{2}-2 \lambda_{3} \mu_{0} \dot{\gamma}+\left(\lambda_{1}-\lambda_{2}\right)\left(\eta+\beta_{3}\right)-2 \mu_{0}\right]
\end{aligned}
$$

$$
\begin{aligned}
\sigma_{2}= & \frac{-\dot{\gamma}^{2}}{2+\left[\left(\lambda_{1}-\lambda_{2}\right) \lambda_{6}+\left(\lambda_{5}-\lambda_{4}\right) \lambda_{3}\right] \dot{\gamma}^{2}+\left(2 \lambda_{3}-\lambda_{4}+\lambda_{5}\right) \dot{\gamma}} \\
& \times\left\{2\left[\left(\eta+\beta_{3}\right) \lambda_{1}-\mu_{0}\right]-\left[\left(2 \lambda_{3}+\lambda_{4}+\lambda_{5}\right) \mu_{0}\right.\right. \\
& \left.-\left(\lambda_{1} \lambda_{5}-\lambda_{2} \lambda_{4}\right)\left(\eta+\beta_{3}\right)\right] \dot{\gamma} \\
& +\left\langle 2 \lambda_{1} \mu^{*}+\left[\left(\lambda_{1}+\lambda_{2}\right) \lambda_{6}-\left(\lambda_{4}+\lambda_{5}\right) \lambda_{3}\right] \mu_{0}\right\rangle \dot{\gamma}^{2} \\
& \left.+\left(\lambda_{1} \lambda_{5}-\lambda_{2} \lambda_{4}\right) \mu^{*} \dot{\gamma}^{3}\right\}
\end{aligned}
$$

It can be seen from the analytical expression of the first normal stress (38), that the curve in plane $\left(\sigma_{1}, \dot{\gamma}\right)$ has four points of intersection with the axis $\dot{\gamma}$. The first two points of intersection are zero 


$$
\dot{\gamma}_{1}=\dot{\gamma}_{2}=0
$$

And another two non-zero points are determined by the following algebraic equation of shear rate

$$
\left(\lambda_{1}-\lambda_{2}\right) \mu^{*} \dot{\gamma}^{2}-2 \lambda_{3} \mu_{0} \dot{\gamma}+\left(\lambda_{1}-\lambda_{2}\right)\left(\eta+\beta_{3}\right)-2 \mu_{0}=0(40)
$$

Solving the equation two points are obtained for which the first normal stress difference is zero (see Eq.41)

For the second normal stress $\sigma_{2}$, five zero points may be obtained, the first two points are given by

$$
\dot{\gamma}_{1}=\dot{\gamma}_{2}=0
$$

The next three points will be obtained by the following algebraic equation

$$
a_{3} \dot{\gamma}^{3}+a_{2} \dot{\gamma}^{2} a_{1} \dot{\gamma}+a_{0}=0
$$

where

$$
\begin{aligned}
& a_{0}=-2\left[\mu_{0}-\left(\eta+\beta_{3}\right) \lambda_{1}\right] \\
& a_{1}=\left(2 \lambda_{3}+\lambda_{4}+\lambda_{5}\right) \mu_{0}+\left(\lambda_{1} \lambda_{5}-\lambda_{2} \lambda_{4}\right)\left(\eta+\beta_{3}\right) \\
& a_{2}=-\left\{2 \lambda_{1} \mu^{*}+\left[\left(\lambda_{1}+\lambda_{2}\right) \lambda_{6}-\left(\lambda_{4}+\lambda_{5}\right) \lambda_{3}\right] \mu_{0}\right\} \\
& a_{3}=-\left(\lambda_{1} \lambda_{5}-\lambda_{2} \lambda_{4}\right) \mu^{*}
\end{aligned}
$$

The relationship between relaxation times may be determined by analysis of the general character of experimental results [4-6]. In general case three roots may be found from the algebraic equation of third order (42). In order to express the experimental behavior of the LC polymer that the second normal stress changes sign two times the algebraic Eq.42 should be second order. It means that a principle of non-oscillatory normal stress is introduced. Thereby a restriction is introduced which is given as

$$
\lambda_{1} \lambda_{5}-\lambda_{2} \lambda_{4}=0
$$

It can be derived from (37)

$$
\lambda_{4}=\alpha_{k} \lambda_{1}, \lambda_{5}=\alpha_{k} \lambda_{2}, \lambda_{5}-\lambda_{4}=\alpha_{k}\left(\lambda_{2}-\lambda_{1}\right) .
$$

where the $\alpha_{k}$ is called as relaxation factor.

Moreover the curve of the $1^{\text {st }}$ and $2^{\text {nd }}$ normal stress differences $\sigma_{1}, \sigma_{2}$ against $\dot{\gamma}$ have the same points of intersection with the axis, it is necessary that

$$
\lambda_{2}=-\lambda_{1} \text {. or } \lambda_{5}=-\lambda_{4} \text {. }
$$

For the above mentioned conditions (44-45) the Eqs. 34-36 are reduced to

$$
\begin{aligned}
& S_{r r}-\lambda_{1}\left(2 \omega_{r z} S_{r z}-\alpha_{k} \omega_{r z} \sigma\right) \\
&= \mu_{0} \dot{\gamma}^{2}+\sin \theta \cos \theta\left[2 \beta_{1} \sin ^{2} \theta+\left(\beta_{2}+\beta_{3}\right)\right] \dot{\gamma} \\
& S_{z z}+\lambda_{1}\left(2 \omega_{r z} S_{r z}-\alpha_{k} \omega_{r z} \sigma\right) \\
&=-\mu_{0} \dot{\gamma}^{2}+\sin \theta \cos \theta\left[2 \beta_{1} \cos ^{2} \theta+\left(\beta_{2}+\beta_{3}\right)\right] \dot{\gamma} \\
& S_{r z}-2 \lambda_{3} \omega_{r z} S_{r z}+\lambda_{6} \omega_{r z} \sigma \\
&=\eta \dot{\gamma}+\mu_{*} \dot{\gamma}^{3} \\
&+\left[2 \beta_{1} \sin ^{2} \theta \cos ^{2} \theta+\left(\beta_{2} \sin ^{2} \theta+\beta_{3} \cos ^{2} \theta\right)\right] \dot{\gamma}
\end{aligned}
$$

For this case, the $1^{\text {st }}$ and $2^{\text {nd }}$ normal stress differences are reduced to

$$
\begin{aligned}
\sigma_{1}= & \frac{2 \dot{\gamma}^{2}}{1+\lambda_{1}\left(\lambda_{6}-\alpha_{k} \lambda_{3}\right) \dot{\gamma}^{2}+\left(\lambda_{3}-\alpha_{k} \lambda_{1}\right) \dot{\gamma}}, \\
& \times\left[\lambda_{1} \mu^{*} \dot{\gamma}^{2}-\lambda_{3} \mu_{0} \dot{\gamma}+\lambda_{1}\left(\eta+\beta_{3}\right)-\mu_{0}\right] \\
\sigma_{2}= & \frac{-\dot{\gamma}^{2}}{1+\lambda_{1}\left(\lambda_{6}-\alpha_{k} \lambda_{3}\right) \dot{\gamma}^{2}+\left(\lambda_{3}-\alpha_{k} \lambda_{1}\right) \dot{\gamma}} \\
& \times\left[\lambda_{1} \mu^{*} \dot{\gamma}^{2}-\lambda_{3} \mu_{0} \dot{\gamma}+\lambda_{1}\left(\eta+\beta_{3}\right)-\mu_{0}\right]
\end{aligned}
$$

For the following special case

$$
\lambda_{3}=0, \lambda_{6}=\lambda, \lambda_{1}-\lambda_{2}=2 \lambda, \mu^{*}=0, \alpha_{k}=0
$$

the Eqs.38-39, as well as Eqs.49-50 are reduced to the results of co-rotational Oldroyd B model [2,21,22].

For Figs 1-3 the material constants are given as

$$
\begin{aligned}
& \lambda_{1}=0.8, \lambda_{3}=0.72, \lambda_{6}=0.8, k=-0.2 \\
& \beta_{3}=2.5, \mu_{0}=32.4, \mu^{*}=0.5
\end{aligned}
$$

Case 2: Director is vertical to flow direction: $\sin \theta=1$, $\cos \theta=0$

$$
\begin{aligned}
& \dot{\gamma}_{3,4}= \\
& \frac{2 \lambda_{3} \mu_{0} \pm \sqrt{4 \lambda_{3}^{2} \mu_{0}^{2}-4\left[\left(\lambda_{1}-\lambda_{2}\right)^{2}\left(\eta+\beta_{3}\right)-2 \mu_{0} \mu^{*}\left(\lambda_{1}-\lambda_{2}\right)\right] \mu^{*}}}{2\left(\lambda_{1}-\lambda_{2}\right) \mu^{*}}
\end{aligned}
$$




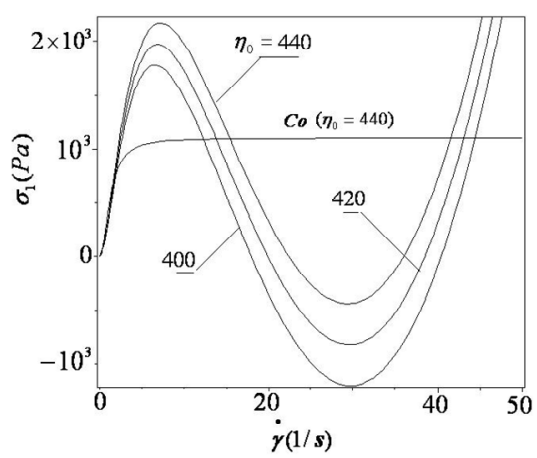

Figure 1. First normal strss difference vs shear rate with variation of viscosity (director parallel to flow direction).

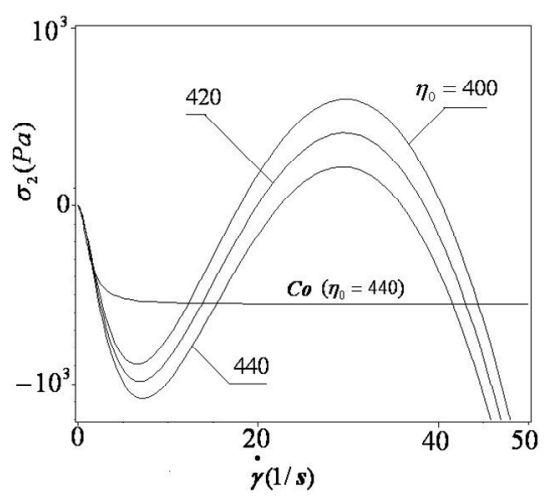

Figure 2. Second normal strss difference vs shear rate with variation of viscosity (director parallel to flow direction).

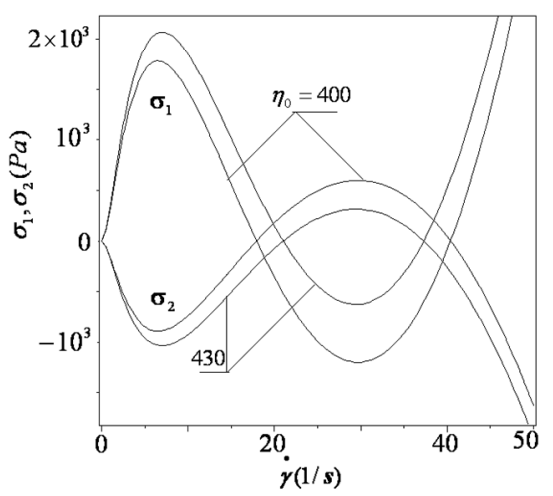

Figure 3. First and second normal stress differences vs shear rate with variation of viscosity $\eta_{0}$ (director parallel to flow direction).

For this case the first and second normal stress differences can be similarly reduced.

In conclusion the following relationships given by (43) to (45) are important

$$
\lambda_{2}=-\lambda_{1}, \quad \lambda_{5}=-\lambda_{4}, \lambda_{4}=\alpha_{k} \lambda_{1}, \lambda_{5}=-\alpha_{k} \lambda_{1}
$$

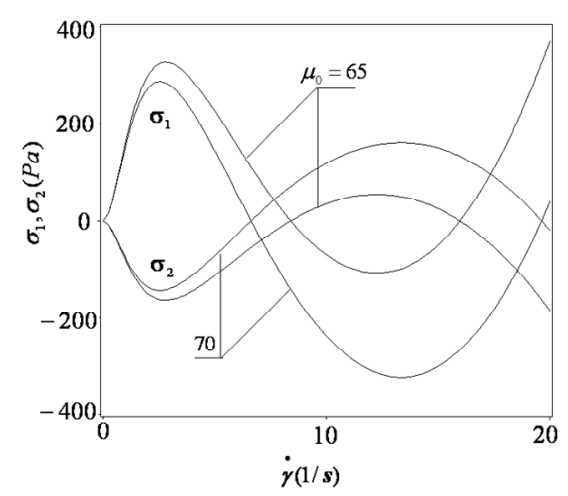

Figure 4. First and second normal stress differences vs shear rate with variation of redartation time $\mu_{0}$ (director parallel to flow direction).

For the constitutive equation the following 8 independent material functions are introduced

$$
\lambda_{1}, \lambda_{3}, \lambda_{6}, \alpha_{k}, \mu_{0}, \mu^{*}, \eta_{0}, \beta_{2}
$$

For Fig. 4 : $\lambda_{1}=0.8, \lambda_{3}=0.45, \lambda_{6}=0.8, k=-0.1$

$$
\beta_{3}=2.5, \mu^{*}=1.50, \eta_{0}=280
$$

\section{UNSYMMETRIC SHEAR STRESS FOR SHEAR FLOW}

For the flow of the LC polymer fluid in circular tube with radius of $\mathrm{R}$, the velocity and director fields of which are are given by (31) and (32), unsymmetric shear stresses will be studied by using the constitutive Eq.24 for the shear-unsymmetric stress action.

Noting $n_{k} n_{s} \omega_{k s}=0$, the right side of (24) are reduced. For the velocity and director vector fields (31) and (32), the constitutive equation in components (24) can be reduced to the following form

$$
\begin{aligned}
& S_{r r}^{s}-\lambda_{1}^{s} \omega_{r z}\left(S_{r z}^{s}+S_{z r}^{s}\right)=\beta_{5} \sin \theta \cos \theta \omega_{r z}, \\
& S_{z z}^{s}-\lambda_{1}^{s} \omega_{z r}\left(S_{r z}^{s}+S_{z r}^{s}\right)=\beta_{5} \sin \theta \cos \theta \omega_{z r}, \\
& S_{r z}^{s}-\lambda_{2}^{s}\left(\omega_{r z} S_{z z}^{s}+\omega_{z r} S_{r r}^{s}\right)=\left(\beta_{4}-\beta_{5} \sin ^{2} \theta\right) \omega_{r z}, \\
& S_{z r}^{s}-\lambda_{3}^{s}\left(\omega_{r z} S_{z z}^{s}+\omega_{z r} S_{r r}^{s}\right)=\left(\beta_{4}-\beta_{5} \cos ^{2} \theta\right) \omega_{z r} \\
& \beta_{4}=\chi_{1}+\chi_{3}, \beta_{5}=\chi_{1}+\chi_{2}, \lambda_{1}^{s}=\lambda_{11}^{s}=\lambda_{22}^{s}, \lambda_{2}^{s}=\lambda_{33}^{s}, \\
& \lambda_{3}^{s}=\lambda_{44}^{s}, \lambda_{i k}^{s}=0 \text {, when } i \neq k .
\end{aligned}
$$

Solving Eqs. 51-54 by the computational symbolic manipulation, such as Maple, more general analytical expressions are obtained by the constitutive equation for the the shear-unsymmetric stresses. The first and second apparent viscosities caused by shear-unsymmetric stress action are given as 


$$
\begin{aligned}
& \eta_{1}^{s}=\frac{-1}{2\left[2+\lambda_{1}^{s}\left(\lambda_{2}^{s}+\lambda_{3}^{s}\right) \dot{\gamma}^{2}\right]} \\
& \times\left\{\lambda_{1}^{s} \dot{\gamma}^{2}\left[\left(\beta_{4}-\beta_{5} \cos ^{2} \theta\right) \lambda_{2}^{s}+\left(\beta_{4}-\beta_{5} \sin ^{2} \theta\right) \lambda_{3}^{s}\right],(55)\right. \\
& \left.+2 \lambda_{2}^{s} \beta_{5} \dot{\gamma} \sin \theta \cos \theta+2 \beta_{4}-2 \beta_{5} \sin ^{2} \theta\right\} \\
& \eta_{2}^{s}=\frac{1}{2\left[2+\lambda_{1}^{s}\left(\lambda_{2}^{s}+\lambda_{3}^{s}\right) \dot{\gamma}^{2}\right]} \\
& \times\left\{\lambda_{1}^{s} \dot{\gamma}^{\cdot}\left[\left(\beta_{4}-\beta_{5} \cos ^{2} \theta\right) \lambda_{2}^{s}+\left(\beta_{4}-\beta_{5} \sin ^{2} \theta\right) \lambda_{3}^{s}\right] .(56)\right. \\
& \left.+2 \lambda_{3}^{s} \beta_{5} \dot{\gamma} \sin \theta \cos \theta+2 \beta_{4}-2 \beta_{5} \cos ^{2} \theta\right\}
\end{aligned}
$$

For the shear flow, the apparent viscosity caused by normal-symmetric stress is given as

$$
\begin{aligned}
& \eta_{n}=\frac{1}{1+\lambda_{1}\left(\lambda_{6}-\alpha_{k} \lambda_{3}\right) \dot{\gamma}^{2}+\left(\lambda_{3}-\alpha_{k} \lambda_{1}\right) \dot{\gamma}} \\
& \times\left\{\left(\eta+\beta_{3} \cos ^{2} \theta\right)+\left(2 \beta_{1} \cos ^{2} \theta+\beta_{2}\right) \sin ^{2} \theta\right. \\
& -\alpha_{k} \lambda_{1} \mu^{*} \dot{\gamma}^{3}+\left(\lambda_{6} \mu_{0}+\mu^{*}\right) \dot{\gamma}^{2} \\
& -\alpha_{k} \lambda_{1}\left[\left(2 \beta_{1} \cos ^{2} \theta+\beta_{2}\right) \sin ^{2} \theta+\beta_{3} \cos ^{2} \theta+\eta\right] \dot{\gamma} \\
& \left.+\lambda_{6} \beta_{1} \sin \theta \cos \theta\left(2 \sin ^{2} \theta-1\right) \dot{\gamma}\right\}
\end{aligned}
$$

The following two special cases are interested.

Case 1: Director is parallel to flow direction $\sin \theta=$ $0, \cos \theta=1$. The apparent viscosity caused by normal stress action is obtained

$$
\begin{aligned}
& \eta_{n}^{\prime \prime}=\frac{1}{1+\lambda_{1}\left(\lambda_{6}-\alpha_{k} \lambda_{3}\right) \dot{\gamma}^{2}+\left(\lambda_{3}-\alpha_{k} \lambda_{1}\right) \dot{\gamma}} \\
& \times\left[\left(\eta+\beta_{3}\right)-\alpha_{k} \lambda_{1} \mu^{*} \dot{\gamma}^{3}+\left(\lambda_{6} \mu_{0}+\mu^{*}\right) \dot{\gamma}^{2}-\alpha_{k} \lambda_{1}\left(\eta+\beta_{3}\right) \dot{\gamma}\right]
\end{aligned}
$$

Case 2: Director is vertical to flow direction $\sin \theta=$ $1, \cos \theta=0$

$$
\begin{aligned}
& \eta_{n}^{\perp}=\frac{1}{1+\lambda_{1}\left(\lambda_{6}-\alpha_{k} \lambda_{3}\right) \dot{\gamma}^{2}+\left(\lambda_{3}-\alpha_{k} \lambda_{1}\right) \dot{\gamma}} \\
& \times\left[\left(\eta+\beta_{2}\right)-\alpha_{k} \lambda_{1} \mu^{*} \dot{\gamma}^{3}+\left(\lambda_{6} \mu_{0}+\mu^{*}\right) \dot{\gamma}^{2}-\alpha_{k} \lambda_{1}\left(\eta+\beta_{3}\right) \dot{\gamma}\right]
\end{aligned}
$$

The total first and second apparent viscosities are the sum of those obtained by the normal-symmetric and shear-unsymmetric results. The shear-unsymmetric stress leads to additional normal stress differences. It can be seen from the expressions that for the normal stress differences any principle changes are given by the the additional one.

Material constants for Figures 5-8:

$$
\lambda_{1}=0.5, \lambda_{3}=0.5, \lambda_{6}=0.8, \lambda_{6}=0.8
$$

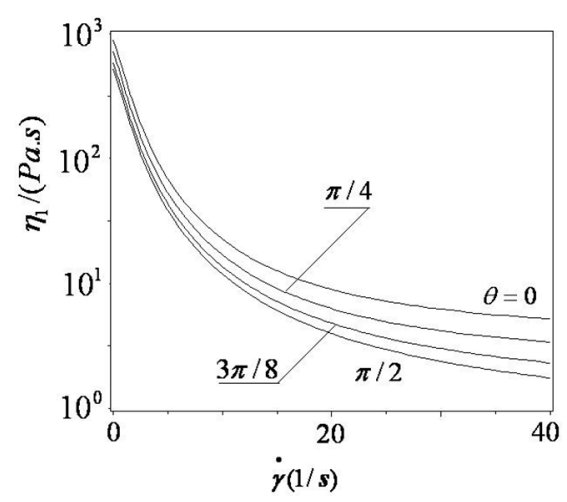

Figure 5. First apparent viscosity vs shear rate (with variation of director vecor).

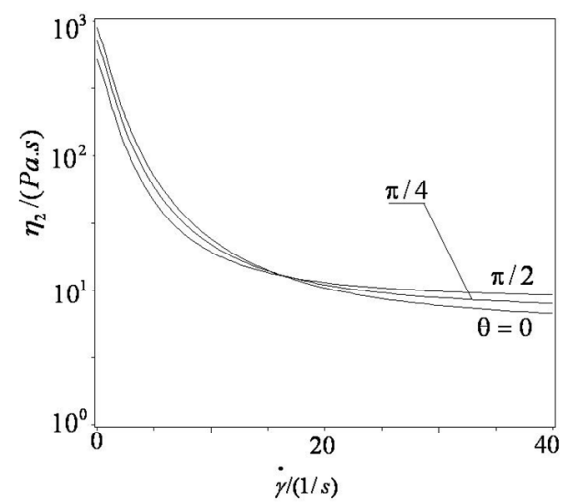

Figure 6. Second apparent viscosity vs shear rate (with variation of director vector).

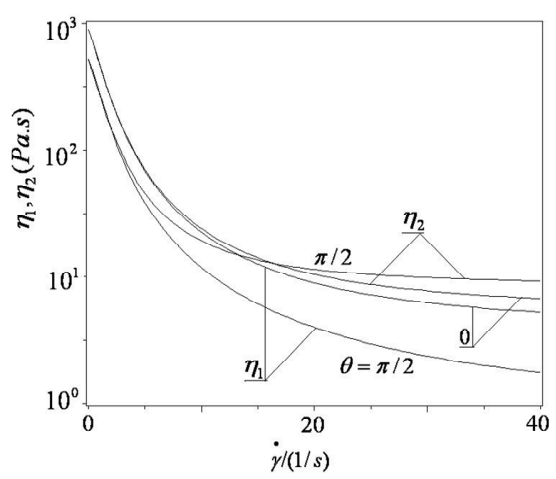

Figure 7. First and second apparent viscosity vs shear rate (with variation of director vector). 


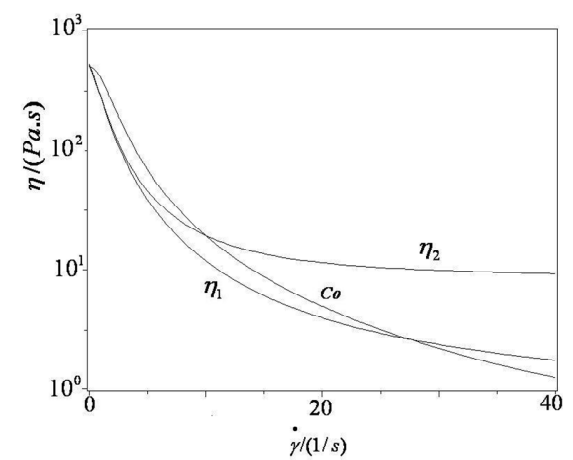

Figure 8. First and second apparent viscosity vs shear rate in comparisom with viscosity calculated by co-rotational Maxwell model (director vector vertical to flow direction). Co.-co-rotational Maxwell model.

$$
\begin{aligned}
& \mu_{0}=1,5, \mu^{*}=1.00, \eta_{0}=500, \beta_{1}=10 \\
& \beta_{2}=20, \beta_{3}=2.5, \beta_{4}=9.5 \beta_{5}=1.0 \\
& \lambda_{1}^{s}=1.0 \lambda^{s}=0.8, \lambda_{3}^{s}=1.0, k=0.01 .
\end{aligned}
$$

\section{DISCUSSIONS AND CONCLUSIONS}

New contribution is given in continuum theory approach to constitutive equation of co-rotational derivative type for the anisotropic viscoelastic fluid-liquid crystalline polymers. A new concept of simple anisotropic fluid is introduced for the fluid. The simple anisotropic fluid is understood as one for which the stress behaviour is assumed to be functional of the whole history of the deformation gradient $F$ and the whole history of spin tensor $W$ instead of rotation tensor $R$ in Green theory. Instead of velocity gradient tensor $D$ in the classic Leslie-Ericksen continuum theory, the stress tensor is described by the deformation gradient $F$ and spin tensor $W$. The components of the stress tensor $S_{i j}$ and its co-rotational derivative are assumed to be a tensor function of $n_{i}, N_{i}, A_{i j}$ and $W_{i j}$ instead of velocity gradient tensor $D$ in classic Leslie-Ericksen continuum theory. Using the tensor analysis approach and analyzing the physical nature of the fluid, a general form of constitutive equation is developed for LC fluid-viscoelastic anisotropic fluid. The rheological behaviour of the normal stress differences is successfully predicted by the model for the LC polymer liquids. The un-symmetry of the shear stress is predicted by the present continuum theory for anisotropic viscoelastic fluid-LC polymer liquids. First and second normal stress differences vs shear rate $\dot{\gamma}$, with variation of viscocity, calculated by the constitutive model are shown by Figure 1 to Figure 3 for director parallel to flow direction. First and second normal stress differences vs shear rate $\dot{\gamma}$ with variation of retardation time is shown by Figure 4. Remarkble difference is seen in Figure 1 and Figure 2 between the results of present anisotropic theory and calculated by isotropic co-rotational Maxwell model. Influence of material constants on normal stress differences is shown by Figure 1 to Figure 3 (viscosity) and Figure 4 (retardation time). It can be seen from the Figure 1 to Figure 4, that the first normal stress difference changes from positive to negative, then to positive, but the second normal stress difference changes from negative to positive, then to negative. In explaining the special behaviour of the normal stress differences the tumbling coefficient $\mu_{*}$ and the relaxation times in the constitutive equation can be considered as fundamental material functions of important influence.

Figure 5 and Figure 6 show the first and second apparent viscosities vs shear rate with variation of director vector respectively. Recognizable change is seen in Figure 5 and Figure 6 for the first and second viscocities with variation of director vector. For comparisom the first and second apparent viscosities vs shear rate with variation of director vector are shown in Figure 7. A remarkable difference between two viscocities is observed by Figure 7. Figure 8 is a plot of the first and second apparent viscosities in comparisom with that calculated by isotropic co-rotational Maxwell model where the director vector is vertical to flow direction.

Firstly, the first and second normal stress differences are successfully predicted by constitutive theory (Figure 1-Figure 4) which is tendentiously in agreement with experimental results of Baek, Larson et al. [4-6] as the experiments were completed with different conditions. Second, two shear stresses and two apparent viscosities are predicted by the present continuum theory (Figure $\mathbf{5}$ -Figure 8), which are in agreement with the theoretical results by Volkov and Kulichikhin [16]. Thereby two shear stresses in the shear flow of LC polymer liquids lead to vortex flow and rotation flow in it. The present phenomenon could be of important practical significance for the modern liquid crystalline display technology.

On basis of the above analysis, the present continuum theory of the constitutive equation of co-rotational type is reasonable and available to predict macroscopic rheological behaviour for this kind of fluids. The present constitutive theory is of fundamental significance on basis of which a series of new anisotropic non-Newtonian fluid problems can be addressed.

\section{ACKNOWLEDGEMENTS}

The present project is supported by National Natural Science Foundation in China: No. 10772177, 19832050. 


\section{REFERENCES}

[1] Han, S.F. (2000) Constitutive equation and computational analytical theory of non-Newtonian Fluids, in Chinese. Science Press, Beijing.

[2] Han, S.F. (2008) Continuum mechanics of anisotropic non-Newtonian fluids-Rheology of liquid crystalline polymer, in Chinese. Science Press, Beijing.

[3] Onogi, S. and Asada T. (1980) Rheology and rheo-optics of polymer liquid crystal. In: Astria, G., Marrucci, G. and Nicolai, Eds., Rheology, Plenum, New York, 127-147.

[4] Baek, S.-G., Magda, J.J. and Larson, R.G. (1993) Rheological differences among liquid-crystalline polymers I. The first and second normal stress differences of PBG solutions. Journal of Rheology, 37(6), 1201-1224.

[5] Baek, S.-G., Magda, J.J., Larson, R.G. and Hudson, S.D. (1994) Rheological differences among liquid-crystalline polymers II. T Disappearance of negative $N_{1}$ in densely packed lyotropic and thermotropes. Journal of Rheology, 38(5), 1473-1503.

[6] Huang, C.M., Magda, J.J. and Larson, R.G. (1999)The effect of temperature and concentration on $N_{1}$ and tumbling in a liquid crystal polymer. Journal of Rheology, 43(1), 31-50.

[7] Doi, M. and Edwards, S.F. (1986) The Theory of Polymer Dynamics, Oxford, London.

[8] Ericksen, J.L. (1960) Anisotropic fluids. Archive for Rational Mechanics and Analysis, 4(1), 231-237.

[9] Ericksen, J.L. (1961) Conservation Laws for Liquid Crystals. Transactions of the Society of Rheology, 5(1), 23-34.

[10] Leslie, F.M. (1979) Theory of flow phenomena in liquid crystals. In: Brown, G.H., Ed., Advances in Liquid Crystals, Academic New York, Vol. 1, pp. 1-81.

[11] Chandrasekhar, S. (1977) Liquid crystals. Cambridge University Press, Cambridge.

[12] Smith, G.F. and Rivlin, R.S. (1957) The anisotropic tensors. Quarterly of Applied Mathematics, Vol. 15, No. 3,
308-314.

[13] Green, A.E. (1964) Anisotropic simple fluid. Proceedings of the Royal Society of London A, 279(1379), 437-445.

[14] Green, A.E. (1964) A continuum theory of anisotropic fluids. Proceedings of the Cambridge Philosophical Society, 60, 123-128.

[15] Volkov, V.S. and Kulichikhin, V.G., (1990) Anisotropic viscoelasticity of liquid crystalline polymers. Journal of Rheology, 34(3), 281-293.

[16] Volkov, V.S. and Kulichikhin, V.G. (2000) Non- symmetric viscoelasticity of anisotropic polymer liquids. Journal of Rheology, 39(3), 360-370.

[17] Larson, R.G. (1993) Roll-cell instability in shearing flows of nematic polymers. Journal of Rheology, 39(2), 175-197.

[18] Han, S.F. (1998) Constitutive equation of Max wellOldroyd type for liquid crystalline polymer and its fluid flow. Proceedings of 3 rd International Conference on Fluid Mechanics, Beijing Institute of Technology Press, Beijing, 723-728.

[19] Han, S.F. (2001) Constitutive equation of liquid crystalline polymer-anisotropic viscoelastic fluid. Acta Mechanica Sinica, in Chinese, 5, 588-600.

[20] Han, S.F. (2004) Constitutive equation of co-rotational derivative type for anisotropic viscoelastic fluid. Acta Mechanica Sinica, 2, 46-53.

[21] Han, S.F. (2007) An unsymmetric constitutive equation for anisotropic viscoelastic fluid. Acta Mechanica Sinica, 2, 46-53.

[22] Han, S.F. (2007) A Constitutive equation of co-ro- tational type for liquid crystalline polymer and influe- nce of orientation on material functions. Journal of Central South University Technology, 14(Suppl 1), 14-18.

[23] Tanner, R.L. (1985) Engineering rheology. Clarendon Press, Oxford.

[24] Zahorski, S. (1982) Mechanics of viscoelastic fluids. Martinus Nijhoff Publishers, The Hague/Boston/London.

[25] Truesdell, C.A. (1951) New definition of a fluid II. The Maxwell fluid. Journal of Pure and Applied Mathematics, 30(9), 115-158. 